\title{
Patient-specific modelling of the cardiovascular system - application to septic shock with a minimal data set
}

\author{
Thomas Desaive $^{1}$, J. Geoffrey Chase ${ }^{2}$, Christina Starfinger ${ }^{2}$, Bernard Lambermont ${ }^{1}$, Alexandre \\ Ghuysen $^{1}$, Philippe Kolh ${ }^{1}$, Pierre C. Dauby ${ }^{3}$, Geoffrey M. Shaw ${ }^{4}$ and Christopher. E. Hann ${ }^{2}$ \\ ${ }^{1}$ Hemodynamics Research Laboratory, University of Liege, Liege, Belgium \\ ${ }^{2}$ Mechanical Eng, Centre for Bio-Engineering, University of Canterbury, Christchurch, New Zealand \\ ${ }^{3}$ Institute of Physics, University of Liege, Liege, Belgium \\ ${ }^{4}$ Department of Intensive Care, Christchurch Hospital, Christchurch, New Zealand
}

\begin{abstract}
We use a previously validated cardiovascular system (CVS) model and parameter identification method to identify the pig-specific parameters during induced endotoxic shock. Six anesthetized healthy pigs weighing 20-30 kg received a $0.5-\mathrm{mg} / \mathrm{kg}$ endotoxin infusion over a period of 30 mins from T0 to T30. Only right heart measurements were obtained and thus significantly less data was available for the model parameter identification compared to previous studies. Errors for the identified model are within $8 \%$ when the model is identified from data, re-simulated and then compared to the clinically measured data. All identified parameter trends match physiologically expected changes. This work represents a further clinical validation for this model-based approach to cardiovascular diagnosis and therapy guidance in monitoring endotoxic disease states.
\end{abstract}

Keywords - cardiovascular system, mathematical model, parameter identification, septic shock.

\section{INTRODUCTION}

Sepsis is a most complex and serious systemic response to infection and has been shown to account for as many deaths in the USA as out-of-hospitals cardiac arrests and four times the number of those who die of breast cancer [1]. More specifically, mortality rates have ranged from $25 \%$ to $80 \%$ over the last few decades [2], making septic shock and multiple organ failure one of the leading causes for morbidity and mortality in the critical care setting.

The CVS model and identification process have already been validated in the identification and model-based analysis of induced endotoxic shock with continuous venovenous hemofiltration (CVVH) therapy [3]. In this research, a porcine model of induced endotoxic shock without hemofiltration is analyzed and the CVS model parameters are identified. The novelty of this identification is that for the first time the identification process is applied to strictly right ventricle signals and no left ventricle signals were measured. This significantly reduced data set is of particular clinical importance, as often only limited data, such as data from only one of the ventricles, is available. The CVS model and identification process are therefore applied here to only the measured right ventricle signals under a series of assumptions. However, similar contractility and afterload trends are obtained when compared to previously reported experimental results [4].

We therefore show the robustness of the methods developed and their potential use to diagnose developing disease states. Hence, this research showcases model-based monitoring and diagnosis of untreated sepsis using further reduced clinical data.

\section{METHODOLOGY}

\section{A. Experimental protocol}

All experimental procedures and protocols used in this investigation were reviewed and approved by the Ethics Committee of the Medical Faculty of the University of Liege. The investigation conforms with the Guide for the Care and Use of Laboratory Animals published by the US National Institutes of Health (NIH Publication No. 85-23, revised 1996).

Experiments were performed on 6 healthy pure pietran pigs of either sex weighing from 20 to $30 \mathrm{~kg}$. The animals received a $0.5 \mathrm{mg} / \mathrm{kg}$ endotoxin infusion (lipopolysaccharide from Escherichia coli serotype 0127:B8; Sigma Chemical, St. Louis, MO, U.S.A.) over 30 mins (from T0 to T30) and received no further intervention. Measurements were obtained for systemic arterial pressure $\left(\mathrm{P}_{\mathrm{ao}}\right)$, pulmonary arterial pressure $\left(\mathrm{P}_{\mathrm{pa}}\right)$ and right ventricle pressure and volume $\left(\mathrm{P}_{\mathrm{rv}}, \mathrm{V}_{\mathrm{rv}}\right)$ as described previously in [4].

Measurements were obtained every 30 minutes into the experiment, from T0 to T300 minutes. Note, that for pig 1, only 4 measurements were obtained (T0 - T90); for pig 2, 9 measurements (T0 - T240); for pig 5, 8 measurements (T0 T210) and for pig 6, 7 measurements (T0 - T180). Pigs 3 and 4 had the full amount of 11 measurements (T0 - T300), thus totalling 50 measurements over all pigs. 


\section{B. CVS model}

The CVS model is a lumped parameter model, previously developed by $[5,6]$ and extended to include one extra compartment for the lung capillaries and a second has been added for the body capillaries (Figure 1). This change separates the venous and arterial systems and resistances, which is more physiologically accurate. Figure 1 shows these modifications with the two new compartments representing the systemic and pulmonary capillaries.

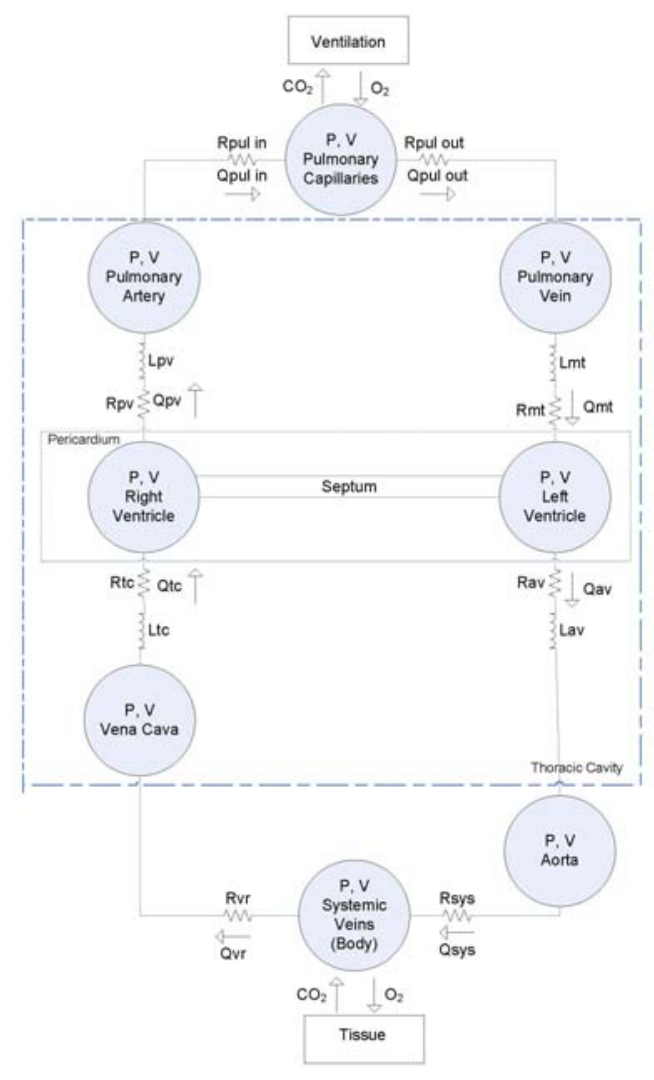

Fig. 1 Closed-loop model of the cardiovascular system

\section{Parameter identification method}

The measured signals for this research that are used for identification are the pressures in the aorta and pulmonary artery $\left(\mathrm{P}_{\mathrm{ao}}, \mathrm{P}_{\mathrm{pa}}\right)$, and the volume in the right ventricle $\left(\mathrm{V}_{\mathrm{rv}}\right)$.

The complete identification process has previously been described [3,7-9], and therefore, only a brief overview is given focusing on the specific aspects of the reduced data set.

First of all, as the left ventricle signals are not measured in this reduced data study, they must be estimated for the identification process to provide an initial starting point.
The left ventricular volume $\left(\mathrm{V}_{\mathrm{lv}}\right)$ is assumed to be the same as the measured right ventricular volume $\left(\mathrm{V}_{\mathrm{rv}}\right)$ for simplicity. The waveforms are artificially generated by scaling a set of previously calculated model outputs to best fit the measured maximum and minimum data values for the pressures and volumes.

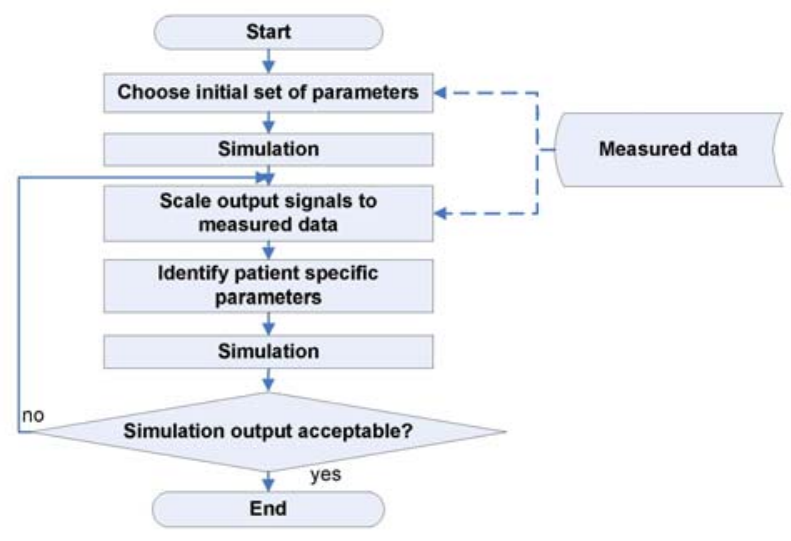

Fig. 2 Parameter identification algorithm

These scaled signals are then used in the parameter identification process, and a new CVS forward simulation is then performed with the identified parameters. The result is a much closer match to the clinical data than what was provided by the first initial parameter set. This animalspecific model simulated output is then compared to the clinical data to assess performance. Subsequently, the output signals are re-scaled and new parameters identified, which are then used to run a further simulation. This iterative process is stopped when the relative error between model output and measured clinical data reaches a set tolerance or fails to improve. Figure 2 gives an overview of the overall identification process.

\section{RESULTS AND DISCUSSION}

Figure 3 illustrates the very good matches achieved for one specific pig in detail at the beginning of the experiment (T0). The upper panel shows the clinical vs. simulated right ventricular pressure and the pressure in pulmonary artery. In the lower panel, the clinical vs. simulated right ventricular volume is shown. It is worth to note, that during the identification process only the systolic (maximum) and diastolic (minimum) values of the measured ventricle volume (EDV, ESV) and arterial pressure (SPAP, DPAP) are used. The right ventricular pressure is not used as this measurement is rarely obtained in a clinical setting. 
However, it can clearly be seen that relatively good matches are nevertheless obtained for the ventricle pressures, even where that measurement was not used in the identification process, further validating the model and identification process. Generally, the errors are well below $10 \%$, which is within typical measurement noise levels.
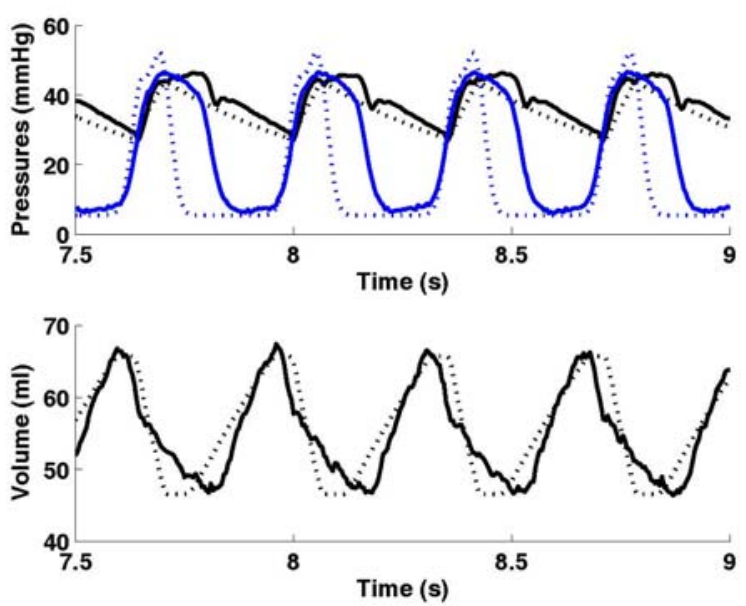

Fig. 3 Model output (dotted) vs clinical (solid line) volume and pressure waveform signals for right ventricle (RV). The upper panel shows the clinical vs. simulated ventricle and arterial pressure. The lower panel shows the clinical vs. simulated ventricle volume.

Figure 4 shows the right ventricular stroke volumes (RVSV) for all identified time segments over all pigs. The solid line represent the clinical data while the crosses represent the CVS model simulation output when re-run using the animal-specific identified model parameters. As can be seen, the model output values match the true clinical values very well, with median absolute percentage errors less than $5 \%$, which is well within measurement or estimate errors $[10,11]$.

The upper panel in Figure 5 shows the clinically measured right ventricular end-systolic elastance ( $\left.E_{\text {esrvf }}\right)$ during the endotoxic shock experiment, as previously reported [4]. The lower panel of Figure 8 shows the similar results obtained from the CVS model and identification process. This elastance, as with all the other model parameters, is identified during the identification process.

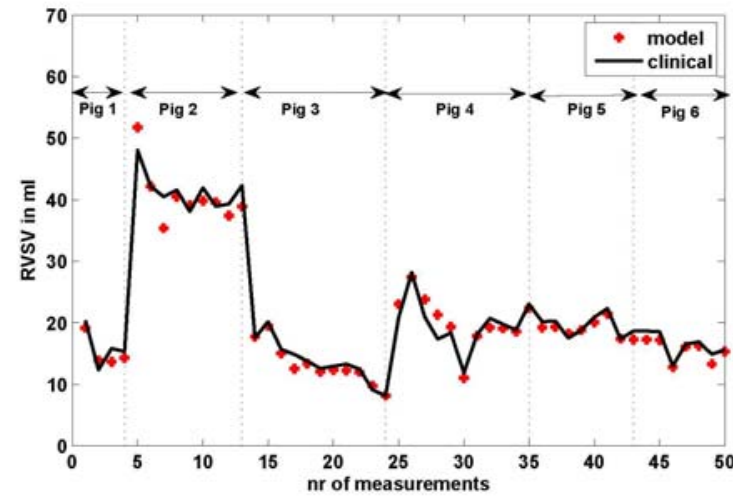

Fig. 4 Clinical (solid line) vs simulated identified animal-specific model (cross) right ventricular stroke volume (RVSV) over all analyzed times and pigs.
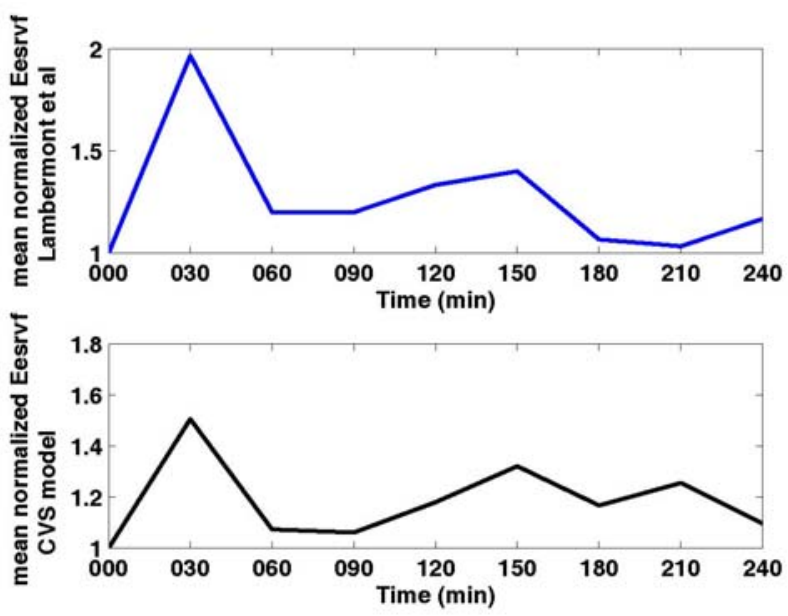

Fig. 5 Mean normalized right ventricular end-systolic elastance $E_{\text {esrvf }}$ over all analyzed pigs during the septic shock experiment. Upper panel: results as obtained by [4], lower panel: results obtained with CVS model and identification process.

\section{CONCLUSIONS}

The integral-based optimization successively identified pig-specific parameters for the extended CVS model using a significantly reduced data set. This shows the ability of the model to adequately and realistically capture the impact of pressure-volume changes during endotoxic shock. In particular, the model is able to aggregate diverse measured data into a clear, clinically and physiologically relevant diagnostic picture as the condition develops. This research thus increases confidence in the clinical applicability and validity of this overall diagnostic monitoring approach preparatory to initial studies with human subjects. 


\section{ACKNOWLEDGMENT}

This work was supported in part by the FNRS (Belgium), the FRST (New Zealand), the University of Liège (Crédit d'Impulsion I-03/21), the Belgian French Community (ARC - Académie Wallonie-Europe) and the University of Canterbury (UoC Targeted Scholarship Scheme).

\section{REFERENCES}

1. Angus DC, and Crowther MA (2003) Unraveling severe sepsis: why did OPTIMIST fail and what's next? JAMA 290: 256-258

2. Angus DC, Linde-Zwirble WT, Lidicker J, Clermont G, Carcillo J, and Pinsky MR (2001) Epidemiology of severe sepsis in the United States: analysis of incidence, outcome, and associated costs of care. Crit Care Med 29: 1303-1310

3. Starfinger C, Chase JG, Hann CE, Shaw GM, Lambermont B Ghuysen A, Kolh P, Dauby PC, and Desaive T (2008) Model-based identification and diagnosis of a porcine model of induced endotoxic shock with hemofiltration. Math Biosci 216: 132--139

4. Lambermont B, Ghuysen A, Kolh P, Tchana-Sato V, Segers P, Gerard P, Morimont P, Magis D, Dogne JM, Masereel B, and D'Orio V (2003) Effects of endotoxic shock on right ventricular systolic function and mechanical efficiency. Cardiovasc Res 59: 412-418
5. Smith BW (2004). Minimal haemodynamic modelling of the heart \& circulation for clinical application. University of Canterbury

6. Smith BW, Chase JG, Nokes RI, Shaw GM, and Wake G (2004) Minimal haemodynamic system model including ventricular interaction and valve dynamics. Medical Engineering \& Physics 26: 131-139

7. Starfinger C, Chase JG, Hann CE, Shaw GM, Lambert P, Smith BW, Sloth E, Larsson A, Andreassen S, and Rees S (2008) Model-based identification of PEEP titrations during different volemic levels. Comput Methods Programs Biomed 91: 135--144

8. Starfinger C, Chase JG, Hann CE, Shaw GM, Lambert P, Smith BW, Sloth E, Larsson A, Andreassen S, and Rees S (2008) Prediction of hemodynamic changes towards PEEP titrations at different volemic levels using a minimal cardiovascular model. Comput Methods Programs Biomed 91: 128--134

9. Starfinger C, Hann CE, Chase JG, Desaive T, Ghuysen A, and Shaw GM (2007) Model-based cardiac diagnosis of pulmonary embolism. Comput Methods Programs Biomed 87: 46-60

10. Baan J, van der Velde ET, de Bruin H, Smeenk GJ, Koops J, van Dijk AD, Temmerman D, Senden J, and Buis B (1984) Continuous measurement of left ventricular volume in animals and humans by conductance catheter. Circulation 70: 812-823

11. Burkhoff D, van der Velde E, Kass D, Baan J, Maughan WL, and Sagawa K (1985) Accuracy of volume measurement by conductance catheter in isolated, ejecting canine hearts. Circulation 72: 440--447 